\title{
PLANIFICACIÓN EDUCATIVA EN CUATRO MUNICIPIOS CHILENOS
}

\author{
ARTURO VALLEJOS ROMERO \\ Profesor e Investigador del Centro de Estudios del Desarrollo Local y Regional \\ de la Universidad de Los Lagos - Osorno, Chile \\ avallejos@ulagos.cl; arturovallejos@yahoo.com
}

\section{DANIELA MONTEIRO TOLEDO}

Profesora de la Universidad Autónoma de Chile - Temuco daniela.monteiro.t@gmail.com

\section{SUSAN ÁVILA ÑIRRIL}

Profesora del Centro de Estudios del Desarrollo Local y Regional de la Universidad de Los Lagos - Osorno, Chile savila@ulagos.cl

\section{RESUMEN}

El artículo presenta una experiencia inédita de planificación educativa municipal participativa y deliberativa en cuatro municipios chilenos de la Región de Los Ríos y Los Lagos. Lo que presentamos es una práctica diferente a la centralizada, no inclusiva y tradicional, que hace hincapié en tres ámbitos, por lo general controvertidos y olvidados en la planificación local: la inclusión de otros actores (aparte de los municipales), la corresponsabilidad en las tareas propuestas y el monitoreo y evaluación compartidos de lo planificado; pilares para la generación de confianzas, una planificación aterrizada y la conformación compartida de un horizonte educacional comunal. Queremos, de fondo, enfatizar que en la toma de decisiones locales "muchos es mejor que pocos" y que estos espacios potencian una planificación austera, pero no menos compleja, de acuerdos colaborativos, que pueda permanecer en el tiempo y generar inclusión y decisiones compartidas en la educación municipal.

PLANIFICACIÓN - PARTICIPACIÓN - MUNICIPIOS - PLANEAMIENTO

\section{ABSTRACT}

EDUCATION PLANING IN FOUR CHILEAN MUNICIPALITIES. The article presents a new experience of participative and deliberative municipal education planning in four Chilean

Este artículo es fruto de la licitación ganada por el Centro de Estudios Regionales - Ceder -, de la Universidad de Lagos, emanada de la Subsecretaría de Desarrollo Regional - Subdere -, para apoyar a cuatro municipios de la Región de Los Ríos y Los Lagos (Panguipulli, Mariquina, Futrono y Frutillar), en la elaboración del Plan de Educación Municipal - Padem. 
municipalities of the Región de los Ríos and de los Lagos. What we presented is a different practice from the centralized one, non inclusive and traditional, that insists on three scopes, generally controverted and forgotten in the local planning: the inclusion of other actors (besides the municipal ones), the joint responsibility in the proposed tasks and the shared monitoring and evaluation of the planned things; pillars for the generation of confidences, for a landed planning and for the shared conformation of a communal educational horizon. We want, basically, to emphasize that in the local decision making "many are better than few" and that these spaces harness an austere planning, but not less complex, in collaborative agreements, that can remain in the long term and generate the inclusion and shared decisions in the municipal education. PLANNING - PARTICIPATION - INCLUSION - MINICIPALITIES

En cualquier ámbito de la intervención social observamos estrategias que tienen como objeto orientar la labor de transformar la sociedad. En esta línea - y para nuestro caso - encontramos al sistema educativo, especialmente en temas relacionados con la planificación nacional, regional y local. Estas estrategias ayudan a enmarcar el procedimiento; a llegar a los objetivos a través de ciertos pasos, que lógicamente van encadenándose para arribar a la elaboración de las acciones concretas que los actores han construido y puesto en operación para alcanzar las metas y objetivos que se han propuesto.

La planificación educacional, especialmente en los gobiernos municipales, ha propiciado formas de tipo más bien normativas, prescriptivas, verticales y centralistas, abiertas e inclusivas a los distintos actores de la comunidad local; esta tarea les cupo a los directores de los Departamentos de Administración de Educación Municipal - Daem -, que con algunos colaboradores más cercanos han propiciado la planificación en los ámbitos locales. Por lo tanto, ésta era una orientación educativa anclada en una visión monolítica y que dependía de la observación que haga la autoridad local.

En esta fórmula encontramos que el gobierno local hace suyos, en una relación uno a uno, todos los problemas educacionales que la autoridad detecte o que la comuna manifieste. No hay en su horizonte la posibilidad de que otros sistemas sociales, como la salud, orden y seguridad, o el sector productivo, entre otros, tengan injerencia en la planeación e implementación de la educación. Si de alguna forma se pudiera esbozar esta posibilidad, las estrategias y metodologías actualmente en uso no dan cuenta de la complejidad que la educación conlleva en la actualidad.

Hablar de planificación con apellido participativo hace alusión a ir más allá de una visión tradicional y jerárquica. Esto no quiere decir que nunca se haya 
incluido a más actores en las iniciativas de planificación local, sino que estamos visibilizando que la planificación con apellido participativo demanda más cosas que informar e informarse, como deliberar y hacerse corresponsable de lo que planificamos cuando somos muchos los que proponemos (Vallejos, 2007).

Este artículo tiene por objeto mostrar la experiencia que, a través del llamado que realizó la Subdere, la Asociación Chilena de Municipalidades ACHM - y el Ministerio de Educación - Mineduc - e a través de invitaciones y licitaciones, el Centro de Estudios del Desarrollo Regional y Local - Ceder -, de la Universidad de Los Lagos, tuvo en cuatro comunas de la Región de Los Ríos y de Los Lagos'. Una propuesta que apoyó la elaboración participativa de la planificación educacional local y que tuvo como fin poder poner en el horizonte de expectativas de los diversos actores comunales la visión, los problemas y posibles soluciones que la educación local trae consigo. La propuesta de intervención que brevemente se mostrará, quiso ir más allá de las formas normativas y regulativas, donde son unos los que diseñan y ejecutan, para poner el énfasis en la coordinación y colaboración, donde fueran los distintos actores de la sociedad local los que pensaran y construyeran sus problemas y soluciones educacionales.

Esta es una experiencia que no tiene como objetivo presentar, a modo recetario, cómo debiera ser la planificación, sino una experiencia que toma en serio el "apellido" participativo y que encuentra luces y sombras, posibilitadores y obstaculizadores. Así también, no es un diseño que sea de una vez y para siempre, sino algo incremental, donde hay que dejar abiertas las posibilidades de que los problemas vuelvan a surgir y para ello hay que volver a coordinarse para enfrentarlos. Creemos que es el devenir de una sociedad altamente contingente y compleja, la cual no se deja tomar a través de modelos que atrapan la realidad; que dominan para llegar a los fines propuestos. Lamentablemente la sociedad nunca fue así; nunca fue como la pensaron los planificadores hace décadas atrás; menos lo es ahora, por lo que planificar es parte del arte de intervenir en una sociedad cada vez más desbocada y con nulas posibilidades de dirigirla desde un centro.

I. Se apoyaron las comunas de Panguipulli, Futrono y Mariquina en la Región de Los Ríos y Frutillar en Los Lagos. 
La exposición contendrá una mirada general al contexto de la planificación en educación, para luego pasar a la planificación municipal y mostrar la oferta participativa del Centro de Estudios del Desarrollo Local y Regional. En un cuarto momento abordaremos los momentos de la observación de los proponentes sobre nudos u obstaculizadores y nodos o posibilitadores que se evidenciaron en la experiencia de planificación local municipal. Por último, cerraremos con algunas reflexiones sobre el proceso realizado.

\section{UNA MIRADA GENERAL AL CONTEXTO DE LA PLANIFICACIÓN EN EDUCACIÓN}

A estas alturas de la modernidad, no hay quienes vaticinen una pronta retirada de la planeación u orientación en general, lo que se extendería a la planificación educacional, constatando que toda directriz impartida por algún organismo público o privado necesita de un mínimo de diseño que ordene los objetivos a cumplir por cualquier actor u organización. En general, las reformas en educación se han hecho evidentes y prioritarias en América Latina por la creciente baja de su calidad, lo que lleva a pensar y mejorar las planificaciones, programas y proyectos que se diseñan e implementan en los diversos sectores del quehacer nacional para ponerlas acorde a los tiempos.

En este empeño se evidencia una crítica a las formas tradicionales de planificación, pues la sociedad ya no es la de antaño y los modelos que sirvieron para controlar y dirigir la sociedad pasada en la actualidad se muestran insuficientes para dar cuenta de una dinámica social mucho más compleja. Esta observación tiene dos aristas que revisten relevancia: la primera nos presenta un cuestionamiento al tipo de planificación y sus ejecutores, es decir, a una planificación de diseño técnico, realizado por técnicos y ejecutada por técnicos. La segunda, si este tipo de intervención muestra falencias, nos preguntamos iquién está interviniendo en una planificación en crisis? Si son técnicos, podemos decir sin temor a equivocarnos: más de lo mismo y escasas posibilidades para salvar sus limitaciones.

La planificación en educación - poniendo un piso temporal no drástico - se remonta a la década de 1960, anclada en las propuestas elaboradas por las teorías del desarrollo económico que se implementaron a mediados del siglo XX. Esta teoría sustentaba que para el desarrollo de los países latinoamericanos era fundamental formar recursos humanos adecuados, que 
respondieran a las demandas del momento y a una región que propiciaba un modelo de industrialización que necesitaba de mano de obra calificada (Sotelo Valencia, 2005; Rostow, 1974; Osorio, 1995; Medina Echavarría, 1967).

En este marco se definió la planificación como una herramienta de intervención, específicamente para orientar cambios. Así, los entes educacionales estatales contarían con funcionarios de carrera y equipos técnicos consagrados a diseñar planes y programas que propiciaran cambios en los procesos educativos. Uno de los puntos claves fue que al levantar estas estructuras de planificación se avanzó en elevar técnicamente el proceso de planificación, generando y ordenando información, levantando diagnósticos de problemas sustentados técnicamente y consolidando una forma de intervención en educación legitimada científicamente. En esta línea se avanzó en identificar posibles problemáticas, pero no se llegó a resultados concretos (Aguerrondo, 1994).

La incapacidad para concretar cambios en la realidad educativa se encontraba en las limitaciones de la propuesta clásica de planificación normativa, que fue incapaz de comprender la realidad social en situaciones de complejidad y conflicto y dirigir desde ese contexto las transformaciones sociales (Aguerrondo, 1994, p.3). Una de las características principales y que marcaba (iy marca!) una planificación normativa era su mirada lineal y no compleja de la realidad, lo que desembocó en estrategias de planificación de cambios educativos fallidos.

En la década de 1990, la alternativa que surge para hacer frente a un modelo de planificación como el descrito fue la planificación estratégica que emergió desde el campo empresarial. Ésta se diferenciaba de la anterior por incorporar la capacidad de reacción frente a la competencia, lo que requería de sistemas de información y monitoreo constantes y atentos a los movimientos de esta última. Aunque esta línea de trabajo se planteó como herramienta que pudiera abordar la problemática social a través de mecanismos que habían dado cuenta de los problemas sociales de buena manera, lo hace y sigue haciendo bajo una perspectiva lineal, ya que se determinaban metas rígidas y que de modo causal - vía esquemas selectivos - priorizaban y modificaban si lo propuesto no llegaba a buen fin.

A principios del siglo XXI, con una sociedad con altos grados de diferenciación, autonomía de sus esferas sociales, con un número cada vez superior de eventos (compleja), dinámica y de alta incertidumbre, se hace aún más necesario planificar, especialmente en educación. La función principal sigue 
siendo organizar el diseño y la implementación de los cambios e innovaciones en un área determinada, pero ello no sucede con la elaboración, donde son unos pocos que observan, diseñan y ejecutan sobre problemas definidos de forma arbitraria y bajo miradas de primer orden, sino con una planeación que tome en cuenta los esquemas de observación de lo que se desea intervenir y de los problemas que se desea solucionar, porque tiene como desafío incorporar, bajo metodologías participativas y deliberativas, a nuevos actores en su proceso. En concreto, el objetivo de este tipo de planificación es fijar metas de forma compartida, además de diseñar y coordinar colaborativamente los medios para lograrlas.

En general, las reformas generales a la educación han traído cambios, los que han tenido sus primeros aprontes de innovación en países desarrollados, pero donde América Latina no se ha quedado atrás. Como ya mencionábamos, la planificación estratégica ha sido uno de estos modelos que principalmente se han usado en modernidades centrales, mientras que en modernidades periféricas, donde encontramos a Chile, se está abriendo la discusión sobre qué modelo sería mejor y más adecuado para la planificación educacional. Uno de estos modelos, cuyo mayor exponente es Carlos Matus (1980, 1989, 1992), es el modelo de planificación situacional o situacional-estratégico, el cual es pensado y elaborado bajo el principio de complejidad social, por lo que las metas y objetivos no pueden ser rígidos, sino que deben posibilitar el adecuarse a las situaciones altamente contingentes de la sociedad moderna.

Resulta interesante observar en esta propuesta que, en primer lugar, hoy no cabe fijarse metas de tipo normativo, más bien se debe acordar una "Imagen-Objetivo" que oriente las decisiones para la acción. Aquí los puntos centrales deben ser acordados socialmente para que la idea matriz de esta orientación de planificación funcione y estos acuerdos se transformen en documentos más amplios que permitan variados puntos de bajadas y no solamente la visión de un planificador. Por lo tanto el planificador tendrá como desafío colaborar en los acuerdos de la Imagen-Objetivo y facilitar su orientación desde una perspectiva de lo posible, dando por sentado que la Imagen-Objetivo se construye a partir de variadas realidades sociales y de observadores que se encuentren para construirla.

En segundo lugar, uno de los problemas centrales en este proceso, y más específicamente en la elaboración del diagnóstico, es el acceso a la información. 
Ello no sólo desde la mirada tradicional de los datos cuantitativos, sino también por medio de la observación y comprensión de las diferentes realidades. Por lo tanto no se trata sólo de describir, sino también de determinar problemáticas y proponer líneas de acción que sean coherentes con las realidades diversas en educación. Se añade a ello el manejo que debe hacerse de la información, situación que actualmente se complica, pues hoy no tenemos problemas para encontrarla y acceder a ella, topándonos con la dificultad de seleccionar la adecuada y relevante para nuestro quehacer. Para este problema está cada vez más vigente la propuesta que desde mediados del siglo pasado nos ha hecho Simon sobre Racionalidad Limitada, donde la consigna es que las carencias y deficiencias que se tiene en la actualidad para procesar la gran complejidad de información que lleve a las decisiones es que se debe hacer uso de decisiones y selecciones de información relevante, como el darles sentido (Torres, Rodríguez, en prensa). Así, el planificador se convierte en un eje articulador entre la investigación científica en educación y su incorporación en la toma de decisiones políticas.

En tercer lugar la implementación deberá orientarse a prever los recursos necesarios para intervenir, haciendo hincapié en una nueva nomenclatura de los recursos necesarios para la planificación, siendo estos materiales (poder hacer), político-culturales (querer hacer) y científicos-técnicos (saber hacer). Se agrega a lo anterior un recurso de suma importancia: el tiempo, por lo que quienes están inmersos en el proceso de planificación deben reconocer los tiempos en que se pueden o no implementar las acciones planificadas.

Por último, y lo más novedoso, es la necesidad de evaluar las acciones diseñadas para observar si las metas propuestas se pueden cumplir o si el plan se está alejando de la Imagen-Objetivo acordada. La evaluación y el monitoreo de una planificación nos da la posibilidad de corregir, redireccionar y modificar los elementos que nos alejen de los objetivos compartidos, ya que de otra manera no sería posible conocer el estado de la intervención y mucho menos generar correcciones.

Esta nueva mirada metodológica de la planificación permite una mayor flexibilidad, de modo que las acciones se articulen en torno a la Imagen-Objetivo acordada. Así, será fundamental reconocer la incidencia de los diferentes actores a la hora de concretar una planificación, por lo que su incorporación en el proceso de diagnóstico, diseño, implementación y evaluación es sumamente 
relevante, especialmente a la hora de legitimar y validar la planeación como instrumento de intervención en educación.

\section{EL PLAN ANUAL DE DESARROLLO DE LA EDUCACIÓN MUNICIPAL: EL CONTEXTO}

Constatamos que la planificación en educación es una práctica relativamente nueva, especialmente en el ámbito de la educación municipal. Ahora, la aseveración anterior podemos de cierto modo relativizarla, diciendo que ésta es un tanto cierta y un tanto errónea. Por lo menos en América Latina, data - como ya lo esbozábamos - desde la década de 1960 del siglo pasado y estaría sustentada en los preceptos de las teorías del desarrollo que apuntaban a la formación de recursos humanos calificados para potenciar el desarrollo de los países de la región. Hasta ese entonces la administración de la educación estaba totalmente en manos del Estado, el cual diseñaba y desarrollaba los planes nacionales. Unos de los ejemplos claros de esta centralidad, la cual podría ser rastreada desde finales del siglo $X I X$ y primera mitad del siglo $X X$, es una visión conservadora y tendiente a la unidad que en Chile fue identificada por el Estado Docente, el cual vertebró su acción bajo los postulados del positivismo latinoamericano (Mascareño, 2000).

En Chile, a principios de la década de 1980 y propiciado por el gobierno militar de la época, se ejecutó una reforma educativa que organizaba la administración de la educación nacional bajo dos aspectos: por un lado el Ejecutivo seguía haciéndose cargo de la política educativa nacional, en lo curricular, lo pedagógico y la asignación de recursos, y por otro lado, la administración de los establecimientos educativos estatales pasó a manos de los gobiernos locales, es decir, los municipios. De esta forma surgió una nueva responsabilidad para los municipios que tenía relación con administrar las escuelas públicas, ahora establecimientos municipales, por lo que debieron comenzar a planificar la educación municipal en su totalidad.

Durante el primer momento se aplicó el paradigma clásico de planificación, donde, más que analizar su estructura, interesaba observar su metodología. La planificación clásica suponía (iy supone!) que los técnicos - en este caso los encargados municipales de educación - planificaban en sus oficinas bajo supuestos técnicos, que no incluían la opinión de los demás actores educativos, 
validándose las propuestas en la lógica interna de los planes. Estos resultaban finalmente documentos técnicos que se formulaban para cumplir la ley, pero que carecían de resultados visibles en el tiempo.

Con la llegada de la democracia a principios de la década de 1990, el gobierno chileno asume el desafío de fortalecer y potenciar la democracia y los espacios democráticos en el país. Este ha sido un proceso paulatino, en el que a través de la modernización del Estado se ha pretendido fortalecer la institucionalidad democrática chilena y donde la educación ha sido un tema relevante, llegándose a la conclusión de que es imperioso propiciar cambios profundos para el desarrollo nacional. En países como los nuestros, la educación es vista como promotora de equidad y como un eje clave para elevar la calidad de vida de la población. Ante los diagnósticos negativos que ésta ha tenido en la actualidad, las bajas puntuaciones que ha obtenido en el concierto mundial y ante la claridad que sin capital humano formado y especializado no hay desarrollo para países como los nuestros, se ha puesto el énfasis en lograr su despegue.

Para el gobierno chileno, lo anterior - entre uno de sus puntos - pasa por fortalecer espacios democráticos, donde no sólo profesionales ligados a la educación sean parte de pensar y planificar lo educativo, sino que se necesita de la participación e inclusión de todos aquellos actores relevantes en el quehacer tanto nacional, como regional y local. Esto, en una primera etapa, dio paso en la década de 1990 a introducir cambios importantes en el ámbito de la planificación de la educación municipalizada, tratando de insertar otras lógicas en el proceso. En este contexto de cambios nace el Plan Anual de Desarrollo de la Educación Municipal - Padem -, emanado de la Ley n. 19.4 I0, cuya finalidad es orientar las actividades educacionales comunales y servir de sustento y/o referente a los planes que cada establecimiento educacional elabore. El Plan tiene como objetivos específicos ordenar y sistematizar las actividades educacionales del municipio a través de los problemas detectados, objetivos propuestos, líneas estipuladas, acciones concertadas y control de lo propuesto por los actores locales de la educación.

Para que un Plan Anual tenga sustento y coherencia, deberá estar sustentado en los lineamientos generales de la Educación Nacional, las Estrategias de Desarrollo Regional y el Plan de Desarrollo Comunal - Pladeco. De ser esto así, la Educación Comunal podrá acoplarse a las demandas y requerimientos que 
la sociedad nacional, regional y local, en un contexto de sociedad global, esté demandando. El cuadro I que se presenta a continuación muestra lo enunciado.

CUADRO I

NIVELES DE PLANIFICACIÓN:

IMPORTANCIA DE LA PLANIFICACIÓN TERRITORIAL EN EDUCACIÓN

\section{POLÍTICA EDUCATIVA NACIONAL}

Educación

pública

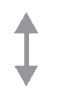

Loce Planes y programas,

Prioridades Política Nacional

POLÍTICA EDUCATIVA REGIONAL

Educación

provincial

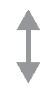

Planes regionales, PAS, GTI

\section{POLÍTICA EDUCATIVA COMUNAL}

Educación

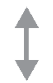

Pladeco y Padem

comunal

PROYECTO EDUCATIVO ESCOLAR

Educación em la

Escuela

PEl, Planes

anuales

ESTUDIANTES - COMUNIDAD

Fuente: Pizarro, 2007.

El Padem fue creado en septiembre del año 1995 mediante la Ley 19.4 I0, que modificó el Estatuto Docente y la Ley de Subvenciones. Su creación obedeció al mejoramiento de la gestión municipal de la educación en temas como las facultades delegadas, los programas de jubilaciones y retiros docentes, y las modificaciones sobre la dotación docente de la educación municipal. Lo anterior se sustentó en las reformas educacionales que ha propiciado el Gobierno de Chile y cuyo objetivo ha sido desarrollar las capacidades locales para la gestión educativa (autogestión) y, a través de procesos participativos, profundizar en la descentralización que se está implementando en pos de modernizar el Estado.

Así, desde su creación, los Daem han ido realizando sus propuestas y concreciones a través de este plan. En este contexto de institucionalización y modernización de la gestión, el Mineduc, en conjunto con la Subdere, desarrolló 
un proceso de difusión y de apoyo a la elaboración de este instrumento, con el objeto de mejorar la capacidad de planificación, gestión, participación y transparencia de la gestión municipal (Pizarro, 2007). El diagnóstico gubernamental observaba una situación desfasada en relación al momento histórico que vivía el país, es decir, al nuevo escenario democrático a que regresaba Chile, y con ello toda América Latina. Además, podemos agregar, pues ello no está explícito en las orientaciones de políticas, la radicalización de una modernidad cada vez más fragmentada y compleja, como variable relevante a tener en cuenta.

Una de las conclusiones a las que se ha llegado, y que funge como situación objetiva de la realidad municipal y su orientación educacional, es que a pesar de incorporar un instrumento de planificación específico para el ámbito educativo, se seguía - y sigue - manteniendo la lógica clásica de planificación, es decir, formular el documento para cumplir con los requerimientos legales, pero no para utilizarlo como una herramienta de gestión. Las evaluaciones arrojan que estos planes se convirtieron en instrumentos que no se validaban entre los actores educativos de cada municipio, pues habría una inexistente participación, siendo estos documentos no compartidos o socializados con la comunidad, lo que generó desconfianzas por parte de los diferentes actores educativos sobre el real efecto que los Padem tendrían en el mejoramiento de la educación municipal.

Es así que durante el año 2007, la Subdere, a través del Departamento de Desarrollo Municipal, que tuvo como socio a la ACHM, propuso y ejecutó la externalización de los Planes a terceros (consultores)². La finalidad fue apoyar

2. La iniciativa piloto tuvo como muestra a 73 municipios. La externalización se configuró bajo dos modalidades; I . a través de una invitación que realizó la ACHM a municipios del país para que sirvieran de apoyo a la elaboración del Padem en algunas comunas y 2. la licitación a través de Chile Compra (Mercado Público) para el resto de las comunas, que estuvo a cargo de la Subdere. En la forma de invitación se incluyó a 29 municipios: 4 en Región de Tarapacá (Camiña, Colchane, Huara y Pica), 6 en Antofagasta (María Elena, Mejillones, Ollague, Sierra Gorda, Tal Tal y Tocopilla), 2 en Coquimbo (Combarbalá y Vicuña), I en la Metropolitana (San José de Maipú), 6 en O’Higgins (Codegua, La Estrella, Litueche, Lolol, Penchaue y Peralillo), 3 en Maule (Chanco, Sagrada Familia y Villa Alegre y ), 4 en Bío Bío (Ñquén y Portezuelo, Quillón y San Pedro), 2 en Araucanía (Victoria y Curacautín) y I en Magallanes (Torres del Paine). En la segunda forma participaron 44 municipios: 4 en la Región Metropolitana (Alhué, Peñalolén, San Pedro y Til Til), 3 en Arica-Parinacota (Arica, General Lagos y Putre), I en Atacama (Diego de Almagro), 4 en Valparaíso (Casablanca, El Quisco, Puchuncaví y Quillota), 2 en O'Higgins (Nancagua y Paredones), 2 en Maule 
la elaboración del Padem a través de un proceso metodológico participativo, que pudiera generar la inclusión de los actores relevantes ligados a la educación municipal y obtener su aprobación de parte de las instancias correspondientes ${ }^{3}$.

En lo operativo, el Padem, según sus contenidos que emanan del Mineduc, debiera presentar un diagnóstico de línea base y uno específico del área, con metas, actividades y programas de acción, un plan de monitoreo y de evaluación para el seguimiento y análisis de las concreciones y no concreciones y el presupuesto de ingresos, gastos e inversión para la ejecución del Plan Educacional Comunal. Esto debiera estar anclado y orientado por las normas técnico-pedagógicas y programas del Ministerio de Educación, así como por las Estrategias de Desarrollo Regional y el Pladeco, para que el sistema educativo, así como el productivo, de salud etc., no se apartaran de las directrices nacionales, regionales y locales de desarrollo.

Recapitulando, es bajo los dos párrafos anteriores, como contexto teórico y operativo, donde la planificación comunal debiera realizarse; en primer lugar según el gobierno -, se debería generar una tendencia participativa planteada en los lineamientos orientadores de planificación, así como en sus instrumentos; en segundo, surgía la posibilidad, a través de las consultorías, de apoyar en los planes que se están ejecutando en cada Departamento de Educación Municipal. En este proceso pudimos observar que la planificación sigue generándose entre "cuatro paredes" y el Padem queda como una obligación, que por lo general se realiza en las semanas anteriores a lo que estipula la ley para su entrega y que posteriormente queda en algún estante, escritorio o "cajón húmedo"

(Colbún y Romeral), 6 en Bío Bío (Alto Bío Bío, Cañete, Lebu, Los Alamos, San Ignacio y Tirúa), 4 en Araucanía (Ercilla, Galvarino, Los Sauces y Saavedra), 9 en Los Ríos (Valdivia, Río Bueno, Panguipulli, Mariquina, Máfil, Los Lagos, Lanco, Lago Ranco y Futrono) y 9 en Los Lagos (Calbuco, Chaitén, Corral, Fresia, Frutillar, Hualaihué, Los Muermos, Maullín y Quemchi).

3. En específico, y según los términos de referencia de la licitación, las líneas fundamentales y específicas que debía contener la iniciativa fueron: a. generar un equipo gestor, con funcionarios del Departamento de Educación Municipal para la elaboración del Padem; b. recopilar la información necesaria, primaria como secundaria, para la elaboración del diagnóstico educativo de la comuna; c. desarrollar el Plan Anual Educativo Municipal, el que debía contener un presupuesto relacionado y un plan de monitoreo y evaluación; d. obtener aprobaciones de las instancias correspondientes, y e. realizar capacitación para transferir conocimientos a los funcionarios municipales. 
de los municipios para el próximo año. En otras palabras, la "ley se obedece, pero no se cumple". Es bajo este contexto y las premisas planteadas donde se enmarca la propuesta del Ceder de la Universidad de Los Lagos.

\section{METODOLOGÍA DE PLANIFICACIÓN PARTICIPATIVA: LA APUESTA DEL CEDER}

La propuesta que la Subdere realizó el año 2007 significó, primero, asumir la participación en su mayor dimensión, es decir, con toda seriedad; segundo, apostar operativamente a una metodología que pudiera dar cuenta de una apuesta que lograra concretarse. Es así como se planteó una iniciativa, que, dada la complejidad actual de la educación, pudiera ser viable, modesta e inclusiva, pero no por ello menos compleja.

La iniciativa consideró tres aspectos fundamentales para dar sustento a la elaboración y concreción de este plan. Estos ejes fueron la participación, lo temático y lo pedagógico.

La variable participativa abogó por la inclusión de los actores educacionales locales que tuvieran relación con la educación municipal y que debían interactuar durante todas las etapas del proceso, desde la conformación del Equipo Gestor hasta el Monitoreo y Evaluación del Plan de Desarrollo. La variable presupuso que los actores pudieran tomar decisiones vinculantes, por lo que participar involucraría manejar información relevante para tomar decisiones en conjunto; a través de una discusión propositiva y creativa, se adoptarían acuerdos que corresponsabilizarían a los actores involucrados.

La variable temática se sustentó en la conformación de una mesa ampliada de trabajo con la participación de todos los actores, así como de pequeñas mesas de discusión ${ }^{4}$, donde se facilitó el diálogo, la identificación de los problemas, la creación de sus soluciones y su concreción en acciones pertinentes para el tiempo planificado. El objetivo último de este eje fue alcanzar un mayor nivel de pertinencia (ampliación de miradas), profundidad y especificidad en el tratamiento de los temas.

4. Para el abordaje metodológico, el trabajo se orientó por la propuesta de los Núcleos de Investigación Participativa - NIP -, propuesto por los profesores Dienel y Harms (2000), Harms y Peneyra (2006) y Harms (2008). 
La variable pedagógica se fundamentó, además de en un alto nivel de compromiso de los actores de la educación en el quehacer local, en un proceso de enseñanza; en un aprender participando y colaborando responsablemente con los demás. En el fondo estuvo la idea de generar procesos de reflexión entre los actores involucrados en el proceso educativo comunal, promoviendo diálogos que convergieran en un aprendizaje mutuo, de los cuales resultaran procesos comunicativos de concertación sobre los puntos a discutir en pos de un objetivo común acordado por todos.

\section{La propuesta metodológica}

La propuesta metodológica se realizó a través de un levantamiento de información primaria y secundaria. El proceso tuvo como finalidad analizar los procesos que no son evidentes a simple vista, articulando el trabajo sobre la base que quienes más conocían sobre la educación comunal eran los propios actores. Bajo la apuesta anterior, el equipo de Apoyo Técnico tuvo un rol orientador y facilitador de las discusiones y opiniones sobre los diferentes temas, pero la decisión final sobre qué se realizaría y qué no fue de exclusividad de los diferentes actores involucrados. Luego de cada reunión, se sistematizó lo trabajado y en cada reunión se recapituló y sancionó lo realizado.

La propuesta conceptual y metodológica se formó y articuló en pos de los retos y dificultades que la participación traía consigo, es decir, decisiones lentas, puntos distantes, intereses distintos, entre muchos otros. No obstante las trabas que observábamos - y observamos - en estos procesos participativos y deliberativos en tradiciones centralistas, asumimos el reto como una oportunidad, donde los actores se hicieran parte del proceso, tomaran decisiones compartidas y se responsabilizaran en las tareas acordadas y a la vez pudieran asumir iniciativas que llevaran a la concepción de ganar mutuamente y no construir relaciones sobre la base de juegos de ganador-perdedor.

En específico, los ejes o pilares estuvieron dados por la propensión al realismo, en función de tópicos como el presupuesto, la realidad local y una relación con la visión macro del resto del territorio (Estrategias de Desarrollo Comunal) y comunal (Plan de Desarrollo Comunal). Asimismo se presentaron y expresaron como fundamentales las responsabilidades en el seguimiento de las líneas estratégicas priorizadas y los acuerdos tomados, para salvar las 
debilidades de toda planificación o política pública, y para que éstas fueran la base de futuras planificaciones que tomaran en cuenta a los actores a través de la participación.

\section{Lo operativo}

Para el trabajo específico, las mesas estuvieron conformadas por distintos actores de la educación municipalizada local, con el objetivo de dialogar, compartir y acordar una visión comunal y generar a través de este proceso mínimos consensos sobre qué querían como educación para sus comunas durante cuatro días de sesiones. Hubo una variedad de actores participantes y relevantes en todos los municipios ${ }^{5}$, los cuales tuvieron como promedio de asistencia 17 actores para los cuatro municipios.

Al ser esta una experiencia nueva en el ámbito de la planificación educacional, pudimos observar elementos interesantes que engranaron como facilitadores y obstaculizadores y que detallamos en el siguiente apartado.

\section{Las buenas, las malas y los desafíos futuros}

Un primer evento que llamó la atención y fue relevante para el trabajo posterior es que, hasta ese momento, aunque existía una visión comunal de educación en el Padem, no se había construido una visión de la educación comunal compartida entre los distintos actores de la educación, lo que generó una discusión rica y productiva. Aunque la mayoría de los participantes había trabajado por mucho tiempo junto y había compartido variadas actividades educativas, ellos no habían puesto sobre la mesa, ni tampoco habían unificado sus concepciones sobre la educación de su localidad. La sorpresa para ellos fue que teniendo como marco general las discrepancias y reparos a la educación

5. Podemos mencionar a Actores Municipales (jefes Daem, de las Secretarías de Planificación Local, entre otros), Organizaciones sociales (juntas de vecinos, organizaciones indígenas, unión comunal de mujeres, entre otros), Organismos públicos (servicios de salud, Carabineros de Chile, entre otros), Representantes políticos (Alcaldes y Concejales), Profesores, Centros de alumnos, Centros de apoderados, Representantes de funcionarios no docentes, Colegios de profesores, Organizaciones económicas (Turismo, entre otros). 
en general, en lo particular concordaban en muchos puntos, lo que facilitó la decisión final sobre la Imagen-Objetivo de la educación comunal.

Uno de los problemas que observamos en el proceso de planeación, pero que no marcó una tendencia generalizada, fue la desconfianza en la pro-

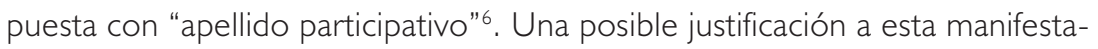
ción expresada tendría su asiento en una tradición de planificación clásica, que diseña, argumenta y ejecuta bajo parámetros técnicos y por lo tanto desconfía de aquellos actores que no tienen esta expertise. El dar paso a espacios deliberativos representó al inicio una tensión para la institucionalidad municipal, pero terminado el proceso nos permitió observar, específicamente en la transferencia metodológica ${ }^{7}$, la aceptación de un proceso que logró aterrizar y transparentar algo que era realizado por unos pocos dejando de lado a muchos. Los actores finalmente plantearon sus inquietudes y dimensionaron la real importancia de un trabajo como el realizado, aduciendo que sus aportes fuesen realmente tomados en cuenta a la hora de implementar o dar cuenta de las actividades y acciones concertadas.

Otro problema observable tuvo que ver con la convocatoria de los actores a ser incluidos en la planificación. La propuesta, reforzada en las reuniones del Equipo Gestor, manifestó la importancia de una convocatoria amplia de actores de la sociedad comunal. Para ello se enunciaron posibles invitados y se dejó, por cercanías y conocimientos, que los Daem realizaran las invitaciones. En algunos municipios se argumentó que la convocatoria fue amplia, pero nos dimos cuenta que no se cumplió el objetivo de la diversidad. Creemos que éste es un punto importante a ser tomado en cuenta para futuras planificaciones locales en educación, ya que la legitimidad del Padem dependerá también de quienes son los llamados a trabajar en él. En esta lógica de planificación clásica, y donde la desconfianza se hace parte del proceso, parece probable que los municipios sigan funcionando bajo una racionalidad burocrática de manutención del control a partir una lógica

6. Este es un evento que no es solamente parte de nuestras regiones, sino que también parte de modernidades centrales. Para un interesante estudio sobre las reservas de los actores políticos y administrativos ante la participación en Europa, ver Pino y Colino (2007).

7. Esta consistió en mostrar la propuesta participativa, es decir, desde cómo se concibió, sus pasos y las herramientas para autoevaluar el proceso. 
de inclusión/exclusión, donde los actores a incorporar debían ser lo menos disonantes para el sistema.

Un factor interesante de visibilizar, y coherente con los puntos de tope que fueron apareciendo desde los actores técnico-políticos, es que en el discurso oficial se manifestaba que la propuesta de un Padem participativo les ayudaría a legitimar y validar las decisiones políticas que se habían tomado en el ámbito educativo comunal, siendo evidente una ruptura de este discurso al tocar el tema del presupuesto municipal para la educación. Los argumentos y respuestas que se exponían iban desde las dilataciones sobre quien debería ser el expositor de tales temas (director del Daem, contador municipal etc.) hasta lo inapropiado de una actividad como ésta, pues podría generar discusiones específicas por demandas financieras irreales de parte de los diferentes actores convocados, a pesar de que en su mayoría fueran cercanos a la línea de gobierno del municipio. Hubo otros municipios que vieron esto como una oportunidad de transparencia para sus arcas fiscales y para hacer visibles los gastos específicos del área educativa y los déficits y problemas a los que se encontraban sometidos.

La consultora externa (Ceder) trató que esto se hiciera viable, pues, primero, no tenía sentido planificar participativamente sin que los actores manejaran información tan relevante como, por ejemplo, con cuántos recursos contaban para poder poner en marcha el Padem; segundo, que un plan o proyecto debe considerar para su viabilidad el tiempo, sus capacidades instaladas y el dinero, este último vital para realizar una planeación aterrizada y que fuese posible cumplir.

En el trabajo específico con los actores, se acordó exponer los ítems más importantes de los recursos financieros y cuál sería el porcentaje asignado para el Padem, para que de esta manera se pudiera definir con mayor precisión qué acciones privilegiarían por sobre otras. Así, esta parte álgida del proceso nos permitió sustentar un plan acorde tanto a dineros reales como a la generación de actividades y acciones que no implicaran dinero, las cuales, para sorpresa de muchos, fueron mayoría en relación a las monetarizadas, como dejar plasmados en la planificación posibles y futuros mecanismos para la obtención de recursos. Esto fue vital, pues nos posibilitó, así como al municipio, presentar información transparente y legitimar el proceso. Para los actores educativos 
fue la oportunidad de conocer información que era relevante para ellos, especialmente para su desempeño cotidiano.

Ante este tipo de dificultades no es posible encontrar fórmulas que determinen cómo podrían éstas ser resueltas exitosamente. Por ello, una de las finalidades en el diseño de planificación participativa propuesto por el Ceder fue el generar confianzas entre los diferentes actores para futuros procesos de planificación que emprendieran autónomamente.

Aunque los problemas fueron planteados como obstáculos, también fueron tomados como oportunidades. En este último punto hubo situaciones que facilitaron el proceso de planeación, donde se pudo observar la idea pensada y verbalizada por las partes, de que una experiencia de este tipo debería ser replicada a niveles micro, especialmente en la elaboración de los Proyectos Educativos Institucionales - PEI - de cada establecimiento educacional de la comuna. En estos proyectos se debería integrar la lógica de una participación deliberativa en la toma de decisiones de las diferentes comunidades educativas, tomando en cuenta que la educación municipalizada atiende a una diversidad de necesidades locales. De esta forma se asume que a partir de un proceso de planificación responsable, informado y deliberativo, cada realidad podría levantar su propio proyecto legitimado por toda la comunidad.

En este punto nos llamó poderosamente la atención que los actores participantes concluyeran que no tendría sentido elaborar un Padem participativo si los PEI siguiesen funcionando con la lógica de la planificación clásica normativa.

Entre los elementos facilitadores del proceso encontramos la seriedad con la que los distintos actores, desde estudiantes primarios y secundarios, profesores, directivos, padres y apoderados, personal paradocente, hasta empresarios y gobierno local, tomaron esta actividad, lo que quedó demostrado no sólo por medio de listas de asistencia, sino también por la participación durante las jornadas y la retroalimentación que generaban para proponer en las sesiones de trabajo.

En algunos municipios fue relevante y vital la participación de grupos indígenas, sobre todo en el caso particular de la región, la etnia Mapuche-huilliche, que ha desarrollado un proceso de vinculación con la educación formal que no ha estado exenta de conflictos, justamente asociados a las características del sistema educativo que impulsa programas nacionales (poco pertinentes al 
mundo indígena) y una planificación normativa (sin flexibilidad para las realidades de las comunidades indígenas). En este sentido, los actores locales participantes (Comuna de Panguipulli), más que presentar una visión negativa, a priori, del proceso, se mostraron atentos, interesados y colaboradores en los distintos momentos del trabajo, destacando que el carácter participativo - dada la fuerza del sentido de inclusión - es positivo, mucho más cuando hasta el momento los procesos de planificación se desarrollaban desde fuera: planificar para ellos y no con ellos.

\section{Evaluación y monitoreo: pata coja de las políticas públicas y la planeación}

Un elemento central que se buscó en la propuesta presentada a la Subdere y a los Gobiernos Locales (Municipios) tenía relación con la Evaluación y Monitoreo del Padem. Como lo mencionamos anteriormente, el punto débil de las planeaciones tradicionales era contar con un sistema de evaluación y monitoreo de las acciones diseñadas. Para este caso nos planteamos que un ejercicio de planificación participativo se podía legitimar y validar a partir de la observación de los resultados obtenidos y los procesos que se vivieron para lograr o no las metas propuestas.

El ámbito del monitoreo y la evaluación estaba directamente relacionado con la verificación de las líneas estratégicas decididas por los diferentes actores. Para esto propusimos una metodología que fuera participativa, dada la necesidad de coherencia interna en la construcción de los distintos Padem. Se planteó la necesidad de que los distintos actores educativos asumieran una participación activa en el Plan de Desarrollo, desde la formulación hasta el momento de monitorearlo y evaluarlo. Para ello se optó por un modelo que fuese llevado a cabo por los participantes y no por una evaluación generada por agentes externos, ya que se ha evidenciado que al ser los propios actores los responsables de evaluar lo que propusieron se logra fortalecer el compromiso de la ciudadanía con el mejoramiento de las políticas educativas de su comunidad.

El enfoque, discutido y acordado por actores educativos, estuvo orientado a generar un proceso de retroalimentación que facilitara la corrección de las fallas en la implementación de las acciones y que a la vez evidenciara las fortalezas del sistema educativo municipal para potenciarlas. El marco para 
dar cuenta de lo implementado tomó el concepto de accountability, es decir, un proceso de control social, en el cual quienes están involucrados en llevar a cabo las actividades deben rendir cuenta de su desempeño y consecuentemente del uso de los recursos asignados a ellos. Los conceptos centrales en un proceso de accountability son la rendición de cuentas por desempeño y la responsabilización por parte de quienes implementan el proceso; esto significa fijar la responsabilidad por las propias acciones y los resultados de dichas acciones (Corvalán, McMeekin, 2006).

Esto se tradujo en un marco de acciones de responsabilidades compartidas, donde todos los actores educativos (técnico, políticos, pedagógicos, directivos, comunitarios, privados) tuvieran responsabilidades y corresponsabilidades que cumplir ante las actividades, acciones y metas autopropuestas en la planificación.

Asimismo, como factor no menos importante, se concluyó que si el diseño no fue importado tampoco sería necesario que su evaluación fuese efectuada por terceros, sino que sus ejecutores y evaluadores serían los propios equipos técnicos municipales, es decir, los responsables directos de ejecutar el proceso y dar cuenta del mismo al finalizar el período que comprende el Plan. Ahora, con esto no se quiso decir que el proceso no tuviera otros responsables directos que colaborarían en el monitoreo de las actividades. En este caso, al haber sido levantado desde una perspectiva participativa, se delegaron responsabilidades a los Directores de los establecimientos educacionales, a los Equipos directivos de los mismos, a los Centros de Padres y Apoderados, a los Centros de Alumnos, y a Organizaciones Gremiales de Trabajadores Paradocentes, entre otros.

Resumiendo, la idea de evaluar y monitorear desde una perspectiva de accountability se refiere a que las responsabilidades no se traspasan a terceros, sino que los mismos actores se hacen corresponsables y asumen que este proceso de planificación no ocurre sólo una vez y para siempre, sino que involucra ir decidiendo, haciendo, volviendo a decidir y rehacer, como un proceso recursivo y constante. Claro está que, para ir más allá de una apuesta como ésta, queda una interesante y vital tarea, que es evaluar como se ha desarrollado el monitoreo y la evaluación por parte de los municipios apoyados. 


\section{UNAS BREVES PALABRAS PARA EL CIERRE}

El diseño y puesta en marcha de una planificación educacional local participativa tiene sus ventajas y desventajas, que han sido esbozadas en el texto. La iniciativa presentada nos deja una clara señal: de que no es posible seguir pensando una planificación prescriptiva y llevada a cabo por unos pocos. La sociedad, aunque sea local, ya no se deja controlar y para gestionarla necesita de la inclusión de los actores sociales. No es posible, debido a muchas falencias de los aparatos administrativos públicos locales (financiamiento, expertise, recursos humanos etc.), querer dar desde sus oficinas respuesta y solución a todos los problemas sociales. Se necesita que de forma colaborativa y coordinada los órganos públicos generen espacios donde la toma de decisiones, la identificación de cuáles son sus problemas, sus posibles soluciones, cuáles serían los modos de implementarlas, hasta observar si se hicieron mal o bien, sean negociadas en espacios que tiendan a incluir a muchos y a hacerse corresponsables de lo que deciden.

La sociedad actual nos impone un nicho organizativo que nos pide elaborar estrategias que privilegien una intervención de tipo reflexiva, donde los modelos propicien la coordinación y la colaboración a través espacios inclusivos y de diálogo abierto. No es raro leer y escuchar que una participación deliberativa tenga altos grados de riesgo y se torne desagradable para aquellos que son actualmente protagonistas (conocedores técnicos y políticos); sin embargo, la experiencia realizada demuestra el valor de la participación en contextos locales, además de las potencialidades que este tipo de acciones entrañan.

Han quedado atrás las planificaciones verticales y normativas, por lo cual esperamos avanzar en esta línea que gana fuerzas en una sociedad moderna.

\section{BIBLIOGRAFÍA}

AGUERRONDO, I. Planificación de la educación hoy en América Latina: ¿Crisis o redefinición? In: REUNIÓN TÉCNICA DE REPLAD/OEA, 6. Ponencia... Campinas: Universidad de Campinas, 1994. (Documento técnico, n. I)

CORVALÁN, J.; MCMEEKIN, R (Ed.). Accountability educacional: posibilidades y desafíos para América Latina a partir de la experiencia internacional. Santiago de Chile: Cide/Preal, 2006. 
DIENEL, P; HARMS, H. Repensar la democracia: los núcleos de intervención participativa. Barcelona: Serbal, 2000.

HARMS, H. La Necesidad de repensar la democracia, 2. Sistema: Revista de Ciencias Sociales, Madrid, n.203-204, p.95-106, May 2008.

HARMS, H.; PEREYRA, S. La Necesidad de repensar la democracia. Sistema: Revista de Ciencias Sociales, Madrid, n. 193, p.3-24, Jul. 2006.

MASCAREÑO, A. La Ironía de la educación en América Latina. Revista Nueva Sociedad, Buenos Aires, n. I65, p. 109- 120, Ener./Febr. 2000.

MATUS, C. Adiós señor presidente. Santiago de Chile: LOM, 1989.

. Planificación de Situaciones. México: Fondo de Cultura Económica, 1980. . Política, planificación y gobierno. Caracas: Fundación Altadir, 1992.

MEDINA ECHAVARRÍA, J. Filosofía, educación y desarrollo. México: Siglo XXI, 1967.

OSORIO, J. Las Dos caras del espejo: ruptura y continuidad en la sociología latinoamericana. México: Triana, 1995.

PINO, E.; COLINO, C. Un Fantasma recorre Europa: renovación democrática mediante iniciativas de promoción de la participación ciudadana en los gobiernos locales (Alemania, Francia, Reino Unido y España). Madrid: CSIC, Sept. 2007. p.33, (Documento de Trabajo, n. 6/7).

PIZARRO, A. El Mineduc y el Padem. In: SEMINARIO DE EVALUACIÓN DE LOS PADEM LICITADOS POR SUBDERE Y ACHM, Santiago de Chile, Dic. 2007. Ponencia... Santiago de Chile: Padem, 2007. Manuscrito

ROSTOW, W. Las Etapas del crecimiento económico, un manifiesto no comunista. México: Fondo de Cultura Económica, 1974.

SOTELO VALENCIA, A. América Latina: de crisis y paradigmas. La teoría de la dependencia en el siglo XXI. México: Plaza y Valdés, 2005.

SUBDERE. Documentos Padem. 2007. Manuscrito.

TORRES, J.; RODRÍGUEZ, D. Introducción a la sociedad de la sociedad. México: Herder. En prensa.

VALLEJOS, A. Políticas públicas, gobiernos locales y resolución de conflictos: cuando en la toma de decisiones "muchos es mejor que pocos". Imaginales: Revista de Investigación Social, Hermosillo, v.3, n.6, p.47-56, Jul./Dic. 2007.

Recebido em: dezembro 2008

Aprovado para publicação em: outubro 2009 